\title{
Corrigendum: Genetically enhanced cows resist intramammary Staphylococcus aureus infection
}

Robert J Wall, Anne M Powell, Max J Paape, David E Kerr, Douglas D Bannerman, Vernon G Pursel, Kevin D Wells, Neil Talbot \& Harold W Hawk

Nat. Biotechnol. 23, 445-451 (2005)

In the abstract on page 445, the quantities given for lysostaphin in the following sentences are incorrect: "To test the feasibility of protecting animals 을 through genetic engineering, transgenic cows secreting lysostaphin at concentrations ranging from 0.9 to $14 \mathrm{mg} / \mathrm{ml}$ in their milk were produced" and "Protection against S. aureus mastitis appears to be achievable with as little as $3 \mathrm{mg} / \mathrm{ml}$ of lysostaphin in milk." The correct quantities are 0.9 to $14 \mu \mathrm{g} / \mathrm{ml}$ and $3 \mu \mathrm{g} / \mathrm{ml}$, respectively. The quantities are correct in the rest of the paper.

\section{Corrigendum: Transient inhibition of BMP signaling by Noggin induces cardiomyocyte differentiation of mouse embryonic stem cells}

Shinsuke Yuasa, Yuji Itabashi, Uichi Koshimizu, Tomofumi Tanaka, Keijiro Sugimura, Masayoshi Kinoshita, Fumiyuki Hattori, Shin-ichi Fukami, Takuya Shimazaki, Hideyuki Okano, Satoshi Ogawa \& Keiichi Fukuda

Nat. Biotechnol. 23, 607-611 (2005)

The order of the authors is incorrect. The correct order is:

Shinsuke Yuasa, Yuji Itabashi, Uichi Koshimizu, Tomofumi Tanaka, Keijiro Sugimura, Masayoshi Kinoshita, Fumiyuki Hattori, Shin-ichi Fukami, Takuya Shimazaki, Satoshi Ogawa, Hideyuki Okano \& Keiichi Fukuda. 